\title{
Avaliação Cognitiva Assistida: Estratégias de Perguntas de Busca de Informação na Resolução de Problemas
}

\author{
Cognitive Assisted Assessment: Strategies of Constraint-Seeking \\ Information Questions in Problems' Resolution
}

\author{
Adriana Aparecida Silvestre Gera* \& Maria Beatriz Martins Linhares \\ Universidade de São Paulo
}

\begin{abstract}
Resumo
O objetivo do estudo foi avaliar indicadores de potencial cognitivo de crianças com queixa de dificuldades de aprendizagem, utilizando procedimento combinado de avaliação psicométrica (Raven) e avaliação assistida (Jogo de Perguntas de Busca com Figuras Diversas - PBFD). Foram avaliadas 34 crianças (8 - 11 anos). O PBFD é delineado em fases, inicial sem ajuda (SAJ), assistência (ASS), manutenção imediata (MAN), manutenção posterior (MANP), transferência I (TRF-I) e transferência II (TRF-II) e avalia desempenho, operações cognitivas e comportamento. Da amostra, $59 \%$ das crianças classificaram-se abaixo da média no Raven. Comparando-se ASS, MAN, MANP, TRF-I e TRF-II em relação a SAJ, verificou-se aumento significativo das perguntas relevantes e do número de acertos (exceção TRF-I). Houve aumento significativo do número de crianças com operações cognitivas positivas em ASS, MAN, TRF-I e TRF-II comparado a SAJ. Os comportamentos positivos ocorreram em pelo menos $62 \% \mathrm{em}$ todas as fases.

Palavras-chave: Avaliação cognitiva; avaliação assistida; dificuldades de aprendizagem.
\end{abstract}

\begin{abstract}
The aim of the study was to assess indicators of the cognitive potential of children with learning disability complaints, using combined procedure of psychometric assessment (Raven) and assisted cognitive assessment (Constraintseeking questions game with several figures - CSQ - SF). Thirty-four learning disabled children were assessed ( $8-11$ years). CSQ - SF is designed by phases, initial with no assistance (SAJ), assistance (ASS), immediate maintenance (MAN), posterior maintenance (MANP), transference I (TRFI) and transference II (TRFII) and it assesses performance, cognitive operations and behavior. In the sample, $59 \%$ of the children were classified below average in Raven. Phases ASS, MAN, MANP, TRF-I and TRF-II in comparison to SAJ shows significant increase of relevant questions and of the number of correct answers (except in the TRFI). There was a significant increase of the number of children with positive cognitive operations in ASS, MAN, TRF-I and TRF-II in relation to SAJ. The positive behaviors occurred in at least $62 \%$ in all phases. Keywords: Cognitive assessment; assisted assessment; learning disabled.
\end{abstract}

As crianças referidas com queixa de dificuldades de aprendizagem constituem-se na clientela de maior proporção na demanda em serviços de saúde e clínicas-escola, quando considerada a faixa etária de sete a 12 anos (Linhares, Parreira, Marturano, \& Sant’Anna, 1993; Peres, 1997).

A Psicopatologia do Desenvolvimento concebe as dificuldades de aprendizagem escolar como uma condição de vulnerabilidade psicossocial da criança ao longo do seu desenvolvimento (Rutter, 1987). De acordo com esta abordagem teórico-conceitual, são vários os fatores que podem influenciar o sucesso ou o fracasso escolar, dependendo da interação que se estabelece entre a criança e seu contex to familiar e social, da história de vida da criança, de seu temperamento e de fatores constitucionais como, por exemplo, o gênero, assim como da fase do desenvolvimento em que se encontra.

Apoio: CAPES / CNPq

* Endereço: Laboratório de Pesquisa em Prevenção de Problemas de Desenvolvimento e Comportamento da Criança (salas 52/53), Av. Tenente Catão Roxo, 2650, Prédio da Saúde Mental, Faculdade de Medicina de Ribeirão Preto, USP, Campus Universitário, Monte Alegre, 14018 900, Ribeirão Preto, SP. linhares@fmrp.usp.br
Diante de crianças com queixa de dificuldades de aprendizagem que buscam ajuda externa à escola na área de saúde, precisam ser estabelecidos critérios para definir a presença ou não de dificuldades e identificar recursos para decidir sobre a necessidade de fato de atendimento extraescolar destas crianças. Recomenda-se que a primeira decisão a ser tomada por profissionais de saúde frente à criança com dificuldades de aprendizagem que busca ajuda especializada seja a realização de um diagnóstico compreensivo. Este deve incluir a avaliação psicológica visando dimensionar os fatores individuais e ambientais envolvidos nas dificuldades enfrentadas pela criança.

No processo diagnóstico, a avaliação cognitiva se insere a fim de identificar o nível intelectual das crianças, assim como dimensionar dificuldades específicas e recursos potenciais do seu funcionamento cognitivo. Esta inclusão justifica-se, pois o insucesso em aprender, embora multideterminado, pode estar vinculado a dificuldades cognitivas generalizadas ou específicas da criança, identificadas no modo como a criança busca, armazena, processa e utiliza informações para resolver questões específicas e problemas relativos à aprendizagem (Rueda, 1996). 
A avaliação diagnóstica na esfera cognitiva tem se baseado, principalmente, na abordagem psicométrica da inteligência (Weiss, 1992). Contudo, além desta, pode-se identificar outra abordagem com enfoque cognitivo, sendo esta subdividida em duas correntes epistemológicas distintas: psicogenética e sócio-cognitiva (Linhares, 1998). No que se refere à abordagem sócio-cognitiva, em particular, visa o conhecimento das estratégias usadas pelos indivíduos durante o processo avaliativo, uma vez que postula que para se chegar a uma avaliação compreensiva, conseqüentemente à prescrição de medidas de intervenção, é necessário conhecer os processos cognitivos e como estes operam na solução de problemas (Coll \& Onrubia, 1993/1996).

O principal pressuposto da avaliação sócio-cognitiva consiste no reconhecimento da plasticidade cognitiva do indivíduo e do caráter de "modificabilidade estrutural cognitiva” decorrente da mediação de aprendizagem (Feuerstein, Rand, Hoffman, Hoffman, \& Miller, 1979b). Assim, na prática de avaliação cognitiva, a avaliação dinâmica assistida, a qual deriva dos pressupostos teóricoconceituais de Vygotsky (1970/1988), surge como uma abordagem promissora na área da avaliação cognitiva, como sugerido nos estudos de Linhares (1995), Linhares, Santa Maria, Escolano, e Gera (1998). Na avaliação cognitiva assistida, as tarefas propostas buscam avaliar tão diretamente quanto possível o funcionamento dos processos psicológicos envolvidos no desempenho de resolução de problemas, em vez de inferir sua operação dos produtos de experiência anterior (Campione \& Brown, 1990). As tarefas de resolução de problemas devem permitir a análise de operações cognitivas. Estas tarefas, segundo Short, Cuddy, Friebert, e Schatschneider (1990), envolvem cinco passos relevantes para alcançar a solução, a saber: identificação da natureza do problema; geração de alternativas de solução (plano); seleção e implementação da melhor solução; monitoramento da efetividade da solução; feedback sobre a solução.

A avaliação cognitiva assistida é uma avaliação dinâmica e interativa, que inclui a intervenção de ensino promovida por um examinador mais experiente durante o processo de avaliação (Linhares et al., 1998). Caracteriza-se por ser um conjunto de estratégias instrucionais utilizadas durante o processo de avaliação, a fim de garantir o fornecimento de ajuda, melhorando as condições da situação de avaliação, para que a criança possa revelar seu desempenho potencial e possa atingir um grau crescente de autonomia em situações de resolução de problemas (Linhares, 1995). Avalia o quanto às crianças podem evoluir em suas estratégias de resolução de problemas frente à "otimização" da situação de avaliação pela examinadora, através de suporte de ajuda instrucional, temporário e ajustável às necessidades da criança.

Embora haja diferenças entre as avaliações psicométrica e assistida, há uma tendência de usá-las de forma combinada, reunindo as informações específicas advindas de cada técnica de modo complementar (Brown \& Ferrara, 1985; Linhares, 1996; Santa Maria \& Linhares, 1999; Swanson, 1995). A avaliação assistida tem sido recomendada para as crianças que apresentam dificuldades de aprendizagem, deficiências cognitivas ou desvantagens culturais (Campione, 1989; Linhares et al., 1998).

O objetivo do presente estudo consiste em avaliar indicadores cognitivos de crianças com queixa de dificuldades de aprendizagem, referidas para atendimento psicológico na área da Saúde, através da avaliação cognitiva assistida associada com a avaliação psicométrica.

\section{Método}

\section{Participantes}

A amostra foi constituída por 34 crianças (denominadas de participantes $-\mathrm{P}$ ou Ps) de 8 a 11 anos, alunos de $1^{\mathrm{a}}$ a $4^{\mathrm{a}}$ série, predominantemente de escolas públicas (82\%) com queixa de dificuldades de aprendizagem, encaminhadas para atendimento psicológico por profissionais da área de saúde para atendimento no Ambulatório de Psicologia Infantil do Hospital das Clínicas da Faculdade de Medicina de Ribeirão Preto - USP. Foram critérios de exclusão a criança apresentar problema neurológico, genético ou psiquiátrico e estar em atendimento para as dificuldades de aprendizagem. O projeto foi aprovado pelo Comitê de Ética em Pesquisa do referido hospital.

\section{Material}

Jogo de Perguntas Busca com Figuras Diversas (PBFD): cujo objetivo era descobrir figuras alvo em um arranjo oito figuras através de perguntas de busca sobre atributos das mesmas (Gera, 2001); Teste de Raven - Escala Especial (Angelini, Alves, Custódio, $\&$ Duarte, 1987); gravador e fitas e termo de consentimento pós-informação.

\section{Procedimento}

Coleta de Dados: A coleta de dados incluiu a realização de três sessões individuais e semanais, que foram gravadas: $1^{\text {a }}$ sessão - teste de Raven; $\mathcal{Q}^{\mathrm{a}}$ sessão - PBFD, fases Preliminar (PRE), Inicial Sem Ajuda (SAJ), de Assistência $(A S S)$ e Manutenção Imediata $(M A N)$; $3^{\text {a }}$ sessão - PBFD, fases Manutenção Posterior (MANP) e Transferência I (TRF-I) e Transferência II (TRF-II). Na fase PRE, fornecia-se um exemplo e as instruções iniciais padronizadas. A fase $S A J$ avaliava os indicadores de desempenho cognitivo real ou de base. A fase $A S S$ avaliava os indicadores de desempenho cognitivo potencial do $\mathrm{P}$ sob condições de ajuda da $\mathrm{E}$, a qual consistia em um suporte instrucional adicional, temporário e ajustável ao desempenho do $\mathrm{P}$, denominado gradiente de mediação. Este era composto por cinco níveis de ajuda, regulados de acordo com a necessidade do P, a saber: feedback; análise comparativa; exemplo de pergunta relevante; retirada dos cartões; demonstração de um modelo do uso da estratégia (Gera, 2001; Linhares et al., 1998). A fase $M A N$ avaliava os indicadores de desempenho cognitivo do $\mathrm{P}$ acerca da manutenção da aprendizagem das estratégias de pergunta de busca de informação com raciocínio de exclusão para solucionar a tarefa realizada, imediatamente após a fase ASS. A fase MANP avaliava os indicadores de desempenho cognitivo do $\mathrm{P}$ acerca da manutenção da aprendizagem das 
estratégias de pergunta de busca de informação após uma semana da fase MAN. A fase $T R F-I$ avaliava indicadores de generalização do desempenho cognitivo do $\mathrm{P}$ das estratégias de pergunta de busca com aplicação direta do papel de perguntar utilizando novos estímulos. Trata-se de situação nova, similar e mais complexa do que as anteriores, utilizando arranjos com maior complexidade dos atributos das figuras. Na fase $T R F-I I$, o objetivo era avaliar indicadores de generalização do desempenho cognitivo do $\mathrm{P}$ das estratégias de pergunta de busca e verificar se ele conseguia responder eficientemente às perguntas do mesmo tipo; o $\mathrm{P}$ e a $\mathrm{E}$ deviam, alternadamente, formular perguntas para descobrir a figura alvo um do outro.

As operações cognitivas utilizadas na resolução da tarefa e o comportamento durante a realização da mesma foram registrados pela $\mathrm{E}$ em protocolos apropriados, de acordo com o sistema de categorias utilizado por Santa Maria e Linhares (1999), Escolano (2000), Gera (2001). As nove categorias de operações cognitivas incluem duas tendências mutuamente exclusivas, caracterizando dois pólos, a saber: "operações cognitivas positivas" e "operações cognitivas negativas”. Os dez comportamentos orientados para a tarefa também foram avaliados em termos de "comportamentos positivos" e "comportamentos negativos".

\section{Análise de Dados}

O teste de Raven foi corrigido de acordo com as normas de padronização brasileira e obtido os percentis. Na avaliação assistida, após as transcrições das gravações, foram analisados: a) indicadores de desempenho cognitivo (Linhares, 1996) - a.1) tipos de perguntas de busca (relevante, irrelevante, incorreta e repetida); a.2) tipos de tentativas de solução (correta, incorreta e correta ao acaso), nas diferentes fases. Os indicadores foram quantificados em termos de taxa ou proporção quanto a: número de perguntas de busca formuladas, tipo de pergunta de busca, número de tentativas realizadas e tipo de tentativas. Baseando-se nesses indicadores de desempenho cognitivo, foi processada a comparação entre a fase SAJ e as demais respectivamente; b) perfis de desempenho cognitivo quanto à manutenção de aprendizagem (Escolano \& Linhares, 1998; Gera, 2001). Primeiramente, com base nos diferentes indicadores de análise, os Ps foram classificados de acordo com os perfis de desempenho cognitivo, os quais basearam-se na manutenção ou não da aprendizagem das estratégias de pergunta de busca de informação (alto-escore, ganhador mantenedor, ganhador dependente da assistência e não ganhador). Em segundo lugar, foram identificados os perfis de desempenho em dois momentos: perfil de desempenho cognitivo envolvendo a fase MAN (avaliava a manutenção da aprendizagem imediata após a assistência da E na fase ASS, e perfil de desempenho cognitivo envolvendo a fase MANP (avaliava o desempenho posterior, após uma semana da aplicação da manutenção imediata). Em terceiro lugar, os dois perfis de desempenho cognitivo (da MAN e da MANP), foram correlacionados entre si, atribuindo-se pontuações de 1 (não ganhador), 2 (ganhador dependente da assistência), 3 (ganhador mantenedor) e 4 (alto-escore); c) perfis de desempenho cognitivo quanto à transferência de aprendizagem (Gera, 2001; Santa Maria \& Linhares, 1999). Procedeu-se uma outra classificação, baseada na generalização ou não do desempenho dos Ps das estratégias de pergunta de busca para uma situação nova, similar e mais complexa do que a anterior nas fases de transferência (TRF-I e TRF-II), classificando-se em: transferidor ou não transferidor. Além disso, os resultados da TRF-I foram correlacionados com os da TRF-II., sendo atribuídas pontuações 1 (transferidores) e zero (não transferidores); d) operações cognitivas e comportamentos orientados para a tarefa. Foi quantificado o número de Ps que apresentava operações cognitivas positivas e comportamentos positivos, comparando-se as diferentes fases com SAJ.

A fim de detectar as diferenças significativas entre o desempenho, as operações cognitivas e os comportamentos positivos na tarefa nas diferentes fases, utilizou-se o teste de Wilcoxon para amostras pareadas, adotando-se o valor de $p \leq 0,05$.

\section{Resultados}

Verificou-se ampla variação quanto ao percentil alcançado no Raven pelos Ps, situando-se desde o percentil 10 ao 95. Uma proporção de 0,59 dos Ps situa-se abaixo do percentil 50 , sendo 0,47 no percentil 25 (definidamente abaixo da média) e 0,12 no percentil 10 (intelectualmente deficiente).

$\mathrm{O}$ restante, distribui-se em 0,20 no percentil 50 (inteligência média), 0,18 no percentil 75 e apenas um $\mathrm{P}$ no percentil 95 .

Observa-se na Tabela 1 que, na fase SAJ, os Ps obtiveram um valor mediano de quatro perguntas por arranjo e formularam mais perguntas relevantes do que não relevantes (irrelevantes, incorretas e repetidas). Em relação às tentativas de solução, os Ps obtiveram valores medianos para o grupo de duas tentativas por arranjo e de uma proporção de 0,56 de tentativas incorretas.

Na comparação entre as fases SAJ e ASS, observa-se que não há mudanças quanto ao número de perguntas de busca por arranjo, no entanto, constata-se um aumento significativo da proporção de perguntas relevantes, atingindo um valor mediano de 0,81 e uma redução significativa das perguntas incorretas. Quanto ao número de tentativas por arranjo, há uma redução significativa de 2 para 1 , sendo que as tentativas corretas aumentam significativamente, atingindo um valor mediano de 0,80. Em contrapartida, as tentativas incorretas e corretas ao acaso diminuem significativamente. Comparando-se a fase SAJ com a fase MAN, após a suspensão da assistência, verifica-se que não há mudança significativa quanto ao número de perguntas de busca por arranjo.

Quanto à relevância das perguntas, constata-se um aumento significativo da proporção de perguntas relevantes de busca de informação, atingindo um valor mediano de o,61. No entanto, há também um aumento da proporção das perguntas irrelevantes e das incorretas, porém em patamares inferiores aos das relevantes, na ordem de 0,15 e 0,12 , 
Gera, A.A.S. \& Linhares, M.B.M. (2006). Avaliação Cognitiva Assistida: Estratégias de Perguntas de Busca de Informação na Resolução de Problemas.

Tabela 1

Indicadores de Desempenho na Resolução do PBFD (Perguntas de Busca e Tentativas de Solução) por Fases (SAJ, ASS, MAN): Mmediana (Md), Amplitude Variação (Min-Max) e Comparação entre as Fases ( $n=34$ )

\begin{tabular}{|c|c|c|c|c|c|}
\hline \multirow[t]{3}{*}{ Indicadores de desempenho PBFD } & \multicolumn{2}{|r|}{ Fases } & \multicolumn{3}{|c|}{ Comparações entre as Fases (valor de $p \leq$ ) } \\
\hline & $\overline{\mathrm{SAJ}}$ & ASS & MAN & \multirow{2}{*}{$\begin{array}{l}\text { SAJ } \\
\text { ASS }\end{array}$} & \multirow{2}{*}{$\begin{array}{l}\text { SAJ } \\
\text { MAN }\end{array}$} \\
\hline & (MD Min-Max) & (MD Min-Max) & (MD Min-Max) & & \\
\hline \multicolumn{6}{|l|}{$\overline{\text { Perguntas de busca }}$} \\
\hline $\mathrm{N}^{\text {o. }}$ de perguntas & $4(1-12)$ & $4(3-5)$ & $4(2-11)$ & 0,25 & 0,56 \\
\hline \multicolumn{6}{|l|}{ Tipos de perguntas (proporção) } \\
\hline Relevante & $0,41(0-1,00)$ & $\begin{array}{l}0,81(0,62- \\
0,96)\end{array}$ & $\begin{array}{l}0,61(0- \\
1,00)\end{array}$ & $<0,001 *$ &, $001 *$ \\
\hline Irrelevante & $0,04(0-0,54)$ & $0,06(0-0,16)$ & $0,15(0-0,31)$ & 0,26 & $0,05 *$ \\
\hline Incorreta & $0,26(0-1,00)$ & $0,11(0-0,29)$ & $0,12(0-1,00)$ & $<0,001 *$ & $0,004 *$ \\
\hline Repetida & $\mathrm{O}(0-0,85)$ & $\mathrm{O}(\mathrm{O}-\mathrm{O}, \mathrm{03})$ & $0,04(0-0,59)$ & 0,26 & 0,32 \\
\hline \multicolumn{6}{|l|}{ Tentativas } \\
\hline $\mathrm{N}^{\circ}$. de tentativas & $2(0-6)$ & $1(1-2)$ & $1-5(0-5)$ & $<0,001^{*}$ & 0,09 \\
\hline \multicolumn{6}{|l|}{ Tipos de Tentativas (proporção) } \\
\hline Correta & $\mathrm{O}(0-1,00)$ & $0,8(0,32-1,00)$ & $0,38(0-1,00)$ & $<0,001 *$ & $0,01 *$ \\
\hline Incorreta & $0,56(0-1,00)$ & $0,16(0-0,68)$ & $0,37(0-0,90)$ & $<0,001 *$ & $0,02 *$ \\
\hline Correta ao acaso & $0,24(0-0,67)$ & $\mathrm{O}(0-0,25)$ & $0,16(0-0,54)$ & $<0,001^{*}$ & $0,03 *$ \\
\hline
\end{tabular}

Nota. ${ }^{*}$ Teste de Wilcoxon $(p \leq 0,05)$

respectivamente. Quanto ao valor mediano de tentativas, este se mantém inalterado. As tentativas corretas aumentam significativamente, atingindo um valor mediano de 0,38, enquanto que as tentativas incorretas e corretas ao acaso diminuem significativamente, atingindo os valores medianos de 0,37 e 0,16 , respectivamente.

Constata-se na Tabela 2 que, na comparação entre as fases SAJ e MANP, não há mudança quanto ao número de perguntas de busca por arranjo. A proporção de perguntas relevantes aumenta significativamente, passando de uma mediana de 0,41 para 0,66. Quanto ao número de tentativas por arranjo, embora haja uma redução do valor das medianas de 2 tentativas para 1, esta não se mostra significativa. A proporção das tentativas corretas aumenta significativamente, atingindo um valor mediano de 0,33, e, em contrapartida, a proporção de incorretas diminui significativamente, atingindo um valor mediano de 0,27.

Comparando-se a fase SAJ e a fase TRF-I, o valor mediano de perguntas de busca mantém-se inalterado. A proporção de perguntas relevantes de busca de informação tende a aumentar, atingindo um valor mediano de 0,66. Quanto ao número de tentativas por arranjo, embora haja uma redução das medianas de 2 tentativas para 1, esta não mostra ser significativa. A proporção de tentativas corretas aumenta significativamente, atingindo um valor mediano de 0,33.

Na comparação entre as fases SAJ e TRF-II, verifica-se que não há mudança significativa em relação ao número médio de perguntas por arranjo. A proporção das perguntas

Tabela 2

Indicadores de Desempenho na Resolução do PBFD (Perguntas de Busca e Tentativas de Solução) por Fases (SAJ, MANP, TRF-I, TRFII): Mediana (Md), Amplitude de Variação (Min-Max) e Comparação entre as Fases ( $n=34$ )

\begin{tabular}{|c|c|c|c|c|c|c|c|}
\hline \multicolumn{2}{|c|}{ Indicadores de desempenho PBFD } & \multicolumn{2}{|l|}{ Fases } & \multicolumn{4}{|c|}{ Comparações entre as Fases (valor de $p \leq$ ) } \\
\hline & SAJ & MANP & TRF-I & TRF-II & \multirow[t]{2}{*}{ SAJ } & \multirow[t]{2}{*}{ SAJ } & \multirow[t]{2}{*}{ SAJ } \\
\hline & $\overline{M d(M i n-M a x}$ & $\operatorname{Md}(\operatorname{Min}-\operatorname{Max})$ & $\operatorname{Md}(\operatorname{Min}-\operatorname{Max})$ & $\overline{M d(M i n-M a x)}$ & & & \\
\hline \multicolumn{8}{|l|}{ Perguntas de busca } \\
\hline $\mathrm{N}^{o}$ de perguntas & $4(1-12)$ & $4(0-12)$ & $4(0-12)$ & $4(2-7)$ & 0,52 & 0,84 & 0,40 \\
\hline \multicolumn{8}{|c|}{ Tipos de perguntas (proporção) } \\
\hline Relevante & $0,41(0-1,00)$ & $0,66(0-1,00)$ & $0,66(0-1,00)$ & $0,69(0-1,00)$ & $0,05^{*}$ & 0,06 & $<0,001^{*}$ \\
\hline Irrelevante & $0,04(0-0,54)$ & $0,1(0-0,51)$ & $0,1(0-0,51)$ & $0,1(0-0,3)$ & 0,19 & 0,68 & 0,61 \\
\hline Incorreta & $0,26(0-1,0)$ & $0,06(0-1,00)$ & $0,06(0-1,00)$ & $0,04(0-0,69)$ & 0,36 & 0,46 & $0,005^{*}$ \\
\hline Repetida & $\mathrm{O}(\mathrm{O}-\mathrm{O}, 85)$ & $\mathrm{O}(\mathrm{O}-\mathrm{O}, 48)$ & $\mathrm{O}(\mathrm{O}-\mathrm{O}, 48)$ & $\mathrm{O}(\mathrm{O}-\mathrm{O}, 36)$ & 0,59 & 0,27 & 0,68 \\
\hline \multicolumn{8}{|l|}{ Tentativas } \\
\hline $\mathrm{N}^{\circ}$ de tentativas & $2(0-6)$ & $1(0-9)$ & $1(0-9)$ & $1(1-4)$ & 0,93 & 0,26 & 0,11 \\
\hline \multicolumn{8}{|c|}{ Tipos de Tentativas (proporção) } \\
\hline Correta & $\mathrm{O}(\mathrm{O}-1,00)$ & $0,33(0-1,00)$ & $0,33(0-1,00)$ & $0,60(0-1,00)$ & $0,02^{*}$ & $0,04^{*}$ & $0,001^{*}$ \\
\hline Incorreta & $0,56(\mathrm{O}-1,00)$ & $0,27(0-0,84)$ & $0,27(0-0,84)$ & $0,20(0-0,73)$ & $0,01 *$ & 0,15 & 0,02 \\
\hline Correta ao acaso & $0,24(0-0,67)$ & $0,18(0-0,47)$ & $0,18(0-0,47)$ & $0,10(0-0,57)$ & 0,10 & 0,85 & $0,008^{*}$ \\
\hline
\end{tabular}


relevantes aumenta significativamente, atingindo um valor mediano de 0,69, e, em contrapartida, a proporção de incorretas reduz significativamente de 0,26 para 0,04. Em relação ao número de tentativas por arranjo, não houve diferença significativa. A proporção das tentativas corretas aumenta significativamente de o para 0,60, e, além disso, a proporção das incorretas e das corretas ao acaso diminui significativamente de 0,56 para 0,20 e de 0,24 para 0,10 , respectivamente. Cabe comentar que um $\mathrm{P}$ não realizou a fase TRF-II por desistência.

Observa-se na Figura 1 que nos valores do perfil de desempenho cognitivo MAN, há um grupo maior de ganhadores (0,88), subdividido em um subgrupo predominante de 0,64 de ganhadores dependentes da assistência (Ps que melhoraram com a assistência presente, mas não mantiveram os ganhos de desempenho após a suspensão da ajuda) e 0,24 de ganhadores mantenedores (Ps que melhoraram seu desempenho na fase ASS e mantiveram o ganho imediatamente após a suspensão da ajuda na fase MAN). Verifica-se ainda que apenas 0,06 dos Ps apresentaram o perfil de alto escore, com bom desempenho logo na fase SAJ, utilizando estratégias eficientes de perguntas relevantes de busca e conseguindo solucionar com sucesso a tarefa, e apenas 0,06 dos Ps apresentaram o perfil de não ganhador.

Observando-se os valores do perfil de desempenho cognitivo MANP, apesar de o grupo de ganhadores continuar sendo predominante $(0,91)$, há uma alteração na distribuição dos Ps nos diferentes perfis, sendo que 0,53 são de ganhadores dependentes da assistência e 0,38 são de ganhadores mantenedores. De um lado, aumenta o número destes últimos e, por outro lado, diminui o número dos primeiros. A proporção de Ps com perfil de alto escore mantém-se inalterada, e em contrapartida, constata-se uma redução de não ganhadores (o,03).
Complementando esses dados, os dois perfis de desempenho cognitivo MAN e MANP não se verificou correlação significativa entre eles; a manutenção dos ganhos de desempenho na fase MAN realizada na mesma sessão da fase ASS não teve correlação com o da MANP, realizada após uma semana.

Com relação ao perfil de desempenho cognitivo quanto à transferência da aprendizagem nas fases TRF-I e TRF-II, encontra-se na amostra apenas 0,21 e 0,35 dos Ps classificados como transferidores, em que esses conseguiram generalizar as estratégias de perguntas relevantes de busca e raciocínio de exclusão de alternativas que levam à solução correta. Nota-se uma leve tendência a haver mais transferidores na situação de TRF-II do que na TRF-I.

Verifica-se na Tabela 3 que há um aumento estatisticamente significativo da freqüência de Ps que apresentaram operações cognitivas positivas, mas fases ASS, MAN, MANP, TRF-I E TRF-II em comparação à fase SAJ. Faz exceção ao aumento a freqüência de Ps relativas às operações cognitivas do tipo conduta reflexiva na fase MAN, encadeamento lógico das questões nas fases MAN e TRF-I, comunicação precisa na fase TRF-I e conduta comparativa na fase TRF-II.

Observa-se na Tabela 4, primeiramente, que a maior parte dos Ps iniciou a tarefa apresentando comportamentos positivos do tipo: sossegado, relaxado, rápido, interessado, participativo, persistente, cuidadoso, disposto e concentrado, com exceção do comportamento do tipo orientado. Em segundo lugar pode-se notar através de comparação da fase SAJ com as demais fases, respectivamente, que há pouca mudança no padrão de comportamentos apresentados pelos Ps, ou seja, estes mantiveram comportamentos positivos durante a realização da tarefa. Alguns comportamentos apresentam modificação significativa quando se compara a fase SAJ com

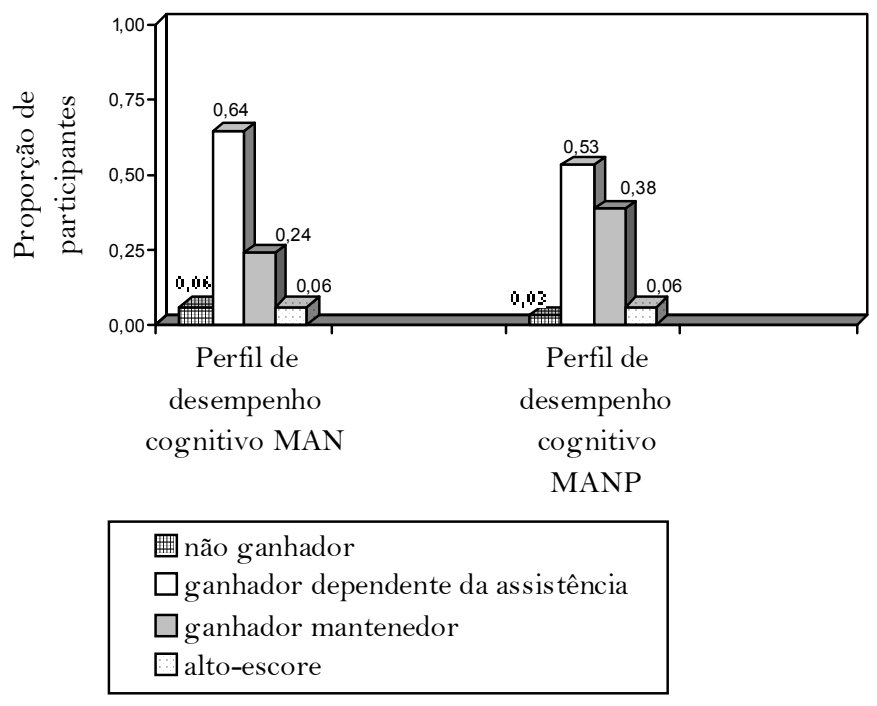

Figura 1. Proporção de participantes com perfis de desempenho cognitivo MAN e MANP na avaliação assistida $(n=34)$. 
Gera, A.A.S. \& Linhares, M.B.M. (2006). Avaliação Cognitiva Assistida: Estratégias de Perguntas de Busca de Informação na Resolução de Problemas.

Tabela 3

Frequiência de Participantes que Apresentaram Operações Cognitivas Positivas nas Fases Inicial Sem Ajuda (SAJ), Assistência (ASS), Manutenção (MAN), Transferência I (TRF-I) e Transferência II (TRF-II) (n=34)

\begin{tabular}{llllll}
\hline Operações cognitivas positivas & SAJ & ASS & MAN & TRF-I & TRF-II \\
\hline Conduta reflexiva & 18 & $30^{*}$ & 22 & $25^{*}$ & $29^{*}$ \\
Percepção clara & 06 & $29^{*}$ & $17^{*}$ & $15^{*}$ & $15^{*}$ \\
Percepção integrativa & 09 & $32^{*}$ & $19^{*}$ & $15^{*}$ & $16^{*}$ \\
Conduta Comparativa & 08 & $30^{*}$ & $19^{*}$ & $18^{*}$ & 13 \\
Identificação de relevância & 05 & $25^{*}$ & $14^{*}$ & $12^{*}$ & $13^{*}$ \\
Encadeamento lógico das questões & 05 & $32^{*}$ & 18 & 16 & $21^{*}$ \\
Auto-correção & 13 & $15^{*}$ & $12^{*}$ & $14^{*}$ & $14^{*}$ \\
Generalização & 06 & $25^{*}$ & $19^{*}$ & $16^{*}$ & $16^{*}$ \\
Comunicação precisa & 14 & $31^{*}$ & $22^{*}$ & 19 & $24^{*}$ \\
\hline
\end{tabular}

Nota. ${ }^{*}$ Teste de Wilcoxon $p \leq 0,05$.

Tabela 4

Freqüência de Participantes que Apresentaram Comportamentos Positivos durante a Realização do PBFD nas Fase Inicial Sem Ajuda (SAJ), Assistência (ASS), Manutenção (MAN), Transferência I (TRF-I) e Transferência II (TRF-II) $(n=34)$

\begin{tabular}{llllll}
\hline Comportamentos positivos & SAJ & ASS & MAN & TRF-I & TRF-II \\
\hline Sossegado & 26 & 26 & 26 & 29 & 29 \\
Relaxado & 34 & 34 & 34 & 34 & 34 \\
Rápido & 21 & 21 & 22 & 24 & 23 \\
Interessado & 34 & 34 & $29 *$ & 34 & 34 \\
Participativo & 32 & 33 & 33 & 34 & 34 \\
Orientado & 09 & $29^{*}$ & $18^{*}$ & $19^{*}$ & $15^{*}$ \\
Persistente & 27 & $32^{*}$ & 29 & $34^{*}$ & $34^{*}$ \\
Cuidadoso & 33 & 32 & 32 & 33 & 33 \\
Disposto & 31 & 30 & 28 & 33 & 34 \\
Concentrado & 28 & 29 & 24 & 32 & 30 \\
\hline
\end{tabular}

Nota. * Teste de Wilcoxon $p \leq 0,05$.

as demais fases. Nas fases ASS, com a ajuda presente, e TRF-I e TRF-II há um aumento significativo no número de Ps que apresentaram os comportamentos do tipo orientado e persistente. Na fase MAN, na qual a ajuda é suspensa, há um aumento significativo em relação aos comportamentos do tipo interessado e orientado. Há aumento significativo do número de Ps com comportamento do tipo orientado na fase ASS em relação a SAJ.

Analisando-se as correlações entre desempenhos cognitivos em MAN, MANP, TRF-I e TRF-II e o percentil no Raven e idade cronológica, foi encontrada apenas a correlação significativa positiva entre desempenho MAN e o percentil no Raven $(r=0,41 ; p \leq 0,05)$. Isso significa que quanto mais alto o percentil do Raven, mais alto o nível de desempenho cognitivo na fase de manutenção do PBFD.

De acordo com a Tabela 5, verifica-se que o perfil desempenho cognitivo MAN correlaciona-se positivamente com o perfil de desempenho cognitivo MANP e TRF-II. Isso indica que quanto melhor o perfil de desempenho cognitivo na fase de manutenção, imediatamente após a assistência, melhor o perfil de desempenho cognitivo avaliado uma semana após a assistência, e na fase de situação nova, semelhante e mais complexa do que as anteriores. Quanto ao perfil de desempenho cognitivo MANP, há correlação significativa positiva do perfil de desempenho cognitivo TRF-I e do TRF-II, quanto melhor o perfil de desempenho cognitivo MANP, melhor o perfil de desempenho cognitivo em ambas as fases de transferência.
Verifica-se correlação significativa positiva entre os perfis de desempenho cognitivo TRF-I e TRF-II; quanto melhor o perfil de desempenho cognitivo na transferência imediata à fase de manutenção envolvendo situação nova, melhor a transferência posterior.

\section{Discussão}

Quanto à avaliação psicométrica, observou-se que 0,59 Ps foram classificados como "intelectualmente abaixo da média” no Raven, predominando a classificação como "definidamente abaixo da média”. Verifica-se, portanto, que a amostra estudada com queixa de dificuldades de aprendizagem, incluía crianças com diferentes níveis intelectuais, constituindo-se em um grupo heterogêneo do ponto de vista cognitivo. Esses achados confirmam os do

Tabela 5

Índices de Correlação de Postos de Sperman (Sr) entre os Desempenhos Cognitivos na Manutenção Imediata (MAN), Manutenção Posterior (MANP), Transferência I (TRF-I) e Transferência II (TRF-II)

\begin{tabular}{lllll}
\hline & MAN & MANP & TRF-I & TRF-II \\
\hline MAN & 1,00 & & & \\
MANP & $0,63^{*}$ & 1,00 & & \\
TRF-I & 0,32 & $0,62+$ & 1,00 & \\
TRF-II & $0,46^{*}$ & $0,82^{*}$ & $0,69^{*}$ & 1,00 \\
\hline
\end{tabular}

Nota. $p \leq 0,05$ 
estudo de Marturano, Loureiro, Linhares e Machado (1997), no qual Ps com dificuldades de aprendizagem apresentam discrepâncias significativas entre a escala verbal e a de execução e em alguns subtestes específicos do WISC.

De forma semelhante, a heterogeneidade do grupo identificada através da medida psicométrica foi verificada através da avaliação assistida, uma vez que alguns Ps revelaram níveis mais elevados de desempenho enquanto outros revelaram níveis mais baixos. Outros estudos, realizados utilizando-se o procedimento de avaliação assistida (Bordin, Linhares, \& Jorge, 2001; Escolano \& Linhares 1998; Santa Maria \& Linhares, 1999) fornecem indicadores do potencial de aprendizagem e permitem detectar diferenciações de padrões individuais. Nesses estudos foram detectadas variações de sinais de recursos potenciais entre as crianças, ou seja, a avaliação assistida possibilitou identificar variações individuais quanto aos recursos cognitivos, diferenciando as crianças quanto à eficiência na solução de problemas, generalização das estratégias eficientes e necessidade de ajuda para atingir soluções adequadas.

Na avaliação cognitiva assistida, realizada através do Jogo PBFD, os Ps, na fase SAJ (desempenho de base), tenderam a formular mais perguntas relevantes do que irrelevantes, porém ao darem a resposta para a solução da tarefa, tenderam a realizar mais tentativas incorretas. Isso demonstra que a maioria dos Ps até conseguia identificar a relevância dos atributos das figuras do arranjo e formular questões, porém não conseguia solucionar a tarefa de maneira correta.

Apesar de grande parte dos Ps terem alcançado a classificação como "definidamente abaixo da média” no Raven, constatou-se que, na avaliação cognitiva assistida, a maioria destas conseguiu melhorar o seu desempenho mediante a assistência presente da examinadora, uma vez que a proporção de perguntas relevantes aumentou e o número de tentativas diminuiu, predominando as tentativas corretas. A “otimização” da situação de avaliação com a ajuda da examinadora permitiu revelar níveis mais elevados de desempenho em situação de resolução de problemas, que se mantiveram mesmo após a suspensão da ajuda. Esta constatação permitiu verificar que os Ps, quando ajudados, podem obter ganhos em seu desempenho e mantê-lo. Esses achados confirmam-se em estudos anteriores (Linhares et al., 1998), nos quais crianças com história de fracasso escolar apresentaram maior eficiência das estratégias de solução com a assistência presente e mesmo após a suspensão desta, sendo observado um aumento de perguntas relevantes e acertos na tarefa. Este fato sustenta a afirmação de que o suporte de ajuda pode favorecer a implementação de estratégias eficientes na resolução de problemas (Santa Maria \& Linhares, 1999; Swanson, 1995).

Analisando-se as variações individuais de desempenho através dos diferentes perfis de desempenho alcançados verificou-se que a possibilidade de se beneficiar da ajuda e reorganizar o padrão de funcionamento cognitivo na resolução da tarefa, variou para cada P. Formaram-se subgrupos diferenciados quanto aos indicadores de eficiência e manutenção de aprendizagem, que sugerem diferentes necessidades educacionais. Os indicadores de desempenho cognitivo, analisados no presente estudo, revelaram subgrupos com diferentes perfis de desempenho, a saber: alto escore, ganhador mantenedor, ganhador dependente da assistência e não ganhador. As diferenças entre os perfis situam-se nas estratégias cognitivas utilizadas para a resolução dos problemas na tarefa do Jogo PBFD.

No perfil de desempenho cognitivo MAN, houve um predomínio de Ps com perfil de ganhador dependente da assistência. Isto significa que os Ps melhoraram o desempenho na fase ASS, mas não mantiveram essa melhora na fase MAN, dependendo da examinadora para obter sucesso na resolução de problemas. O sub-grupo ganhador mantenedor apresentou uma proporção menor de Ps, os quais mantiveram a melhora do desempenho na fase MAN. Os Ps com perfil alto-escore representaram um grupo menor, que manifestaram bom desempenho logo na fase SAJ, assim como o subgrupo não ganhador, que indicaram uma dificuldade maior frente à tarefa.

No perfil de desempenho cognitivo MANP, houve uma redistribuição dos Ps quanto ao perfil. As proporções de Ps com perfil ganhador dependente da assistência e de não ganhador diminuíram, enquanto que a proporção de ganhador mantenedor aumentou. Isso sugere que um número maior de Ps foi capaz de manter a melhora do seu desempenho, mesmo após a suspensão da ajuda, apresentando assim estratégias de busca de informação mais eficientes. A proporção de Ps alto escore manteve-se inalterada.

A variação quanto aos perfis de desempenho cognitivo sugere que o grupo referido com queixa de dificuldades de aprendizagem é heterogêneo, incluindo tanto Ps com recursos quanto Ps com dificuldades. Vale a pena ressaltar ainda que o potencial individual para mudança de cada $\mathrm{P}$ e o quanto de ajuda era necessária para que essa mudança ocorresse são fatores que contribuem para essa variação individual.

Quanto à fase de transferência, que é de maior dificuldade e complexidade no processo de avaliação assistida, uma vez que traz novidade quanto ao material, embora mantenha a mesma natureza do problema já utilizado nas fases anteriores, verificou-se que a maioria dos Ps formulou perguntas relevantes e o número de tentativas diminuiu, predominando as tentativas corretas. Esses indicadores de desempenho dos Ps na fase TRF permitiram revelar dois grupos com diferentes desempenhos, a saber: transferidor e não transferidor. As diferenças entre esses perfis são estabelecidas em relação à generalização dos ganhos de desempenho obtidos durante o processo de avaliação assistida.

No desempenho da fase TRF-I, houve um predomínio de Ps com perfil de não transferidor, isto é, aqueles que apresentaram desempenho inferior ao patamar estipulado em relação às perguntas relevantes de busca e inferior ao patamar estipulado em relação aos acertos na fase TRF. Os Ps restantes foram classificados como transferidores, o que corresponde àqueles que apresentaram patamares acima do 
estipulado nesses indicadores de desempenho. No perfil de desempenho da fase TRF-II, por sua vez, houve uma redistribuição quanto ao perfil. A proporção de Ps com perfil de não transferidor diminuiu e, conseqüentemente, a de transferidor aumentou. Isso sugere que, apesar da situação de resolução de problema ser complexa nesta fase, os Ps generalizaram com mais facilidade. Isto, provavelmente, pode ser atribuído a dois fatores, a saber: a) o fato de que nesta fase o $\mathrm{P}$ e a $\mathrm{E}$ intercalam-se, formulando perguntas de busca de informação, o que sugere que o P passa a contar com modelos de perguntas fornecidos pela $\mathrm{E}$; b) o fato de os Ps já conhecerem os arranjos utilizados nesta fase, uma vez que são os mesmos da fase de TRF-I.

A amostra estudada continuou apresentando heterogeneidade quanto à manutenção e transferência da aprendizagem, uma vez que algumas destas conseguem manter e transferir o repertório aprendido enquanto outras não. Esse achado vêm ao encontro a estudos realizados por Brown e Ferrara (1985) e por Ferrara, Brown e Campione (1986), nos quais verificaram-se variações intra-grupo quanto ao desempenho dos Ps na transferência da aprendizagem.

Segundo Martin e Marchesi (1993/1995), o processo de manutenção e transferência tem clara relação com a inteligência e a aprendizagem, uma vez que se aprendemos algo é porque somos capazes de colocar em funcionamento conhecimentos e estratégias que já possuímos e de aplicálas a uma nova situação. Portanto, transferir é um dos processos que caracteriza o comportamento inteligente. Além disso, estes dois autores salientam que estudos realizados com crianças com problemas de aprendizagem e com crianças com atraso mental mostram justamente este aspecto; essas apresentaram dificuldade de aprender as informações para solucionar problemas com autonomia, assim como dificuldade de generalizar o aprendido. $\mathrm{O}$ insucesso em aprender pode estar vinculado a dificuldades na área cognitiva das crianças, identificadas no modo como estas buscam, armazenam, processam e utilizam informações para resolver questões específicas e problemas relativos à aprendizagem (Ferreti \& Butterfield, 1992; Rueda, 1996).

Para que a criança transfira o aprendido para uma situação semelhante, é necessário que ela estabeleça relações conectando necessidades imediatas com outras necessidades, sempre em expansão. Feuerstein, Rand e Hoffman (1979a), denominaram essas relações de transcendência. Segundo Mentis (1997), a transcendência movimenta o aprendiz para além da necessidade direta e imediata, ampliando o sistema de necessidades do mediado, incluindo a necessidade por compreensão, por pensamento reflexivo e pela formação de relações entre idéias.

Finalizando, os achados da presente pesquisa sugerem que os Ps referidos com dificuldades de aprendizagem apresentaram indicadores de dificuldades em manter e transferir a aprendizagem de estratégias de resolução de problemas, envolvendo perguntas de busca de informação. Contudo, uma vez assistidas durante o processo da aprendizagem podem vir a revelar recursos potenciais de aprendizagem. Os dados, também, sugerem que, para que ocorresse a manutenção do aprendido, pareceu ser necessária a repetição de estímulos em diferentes contextos, uma vez que a maioria das Ps deste estudo melhorou seu desempenho em fases em que houve a repetição de estímulos de arranjos conhecidos e precisou de mediação para realizar a transferência de aprendizagem para novos estímulos.

\section{Referências}

Angelini, A.L., Alves, I.C.B., Custódio, E.M., \& Duarte, W.F. (1987). Matrizes progressivas coloridas: Escala especial-Raven. São Paulo: Casa do psicólogo. (Original publicado em 1947)

Bordin, M.B.M., Linhares, M.B.M., \& Jorge, S.M. (2001). Aspectos cognitivos e comportamentais na média meninice de crianças pré-termo de $\leq 1500$. Psicologia: Teoria e Pesquisa, 17, 49-57.

Brown, AL. \& Ferrara, R.A. (1985). Diagnosing zones of proximal development. In J.V. Wertsch (Ed.), Culture communication and cognition: Vygotskian perspectives (pp.273-305). Cambridge: Cambridge University Press.

Campione, J.C. (1989). Assisted assessment: A taxonomy of approaches and an outline of strengths and weaknesses. Journal of Learning Disabilities, 22, 151-165.

Campione, J.C. \& Brown, A.L. (1990). Guided learning and transfer: implications for approaches to assessment. In N. Fredericksen, R. Glasser, A. Lesgold, \& M.G. Shafto (Eds.), Diagnostic monitoring of skill and knowledge acquisition (pp.141172). New Jersey: Lawrence Erlbaum.

Coll, C. \& Onrubia, J. (1996). Inteligência aptidões para a aprendizagem e rendimento escolar (A.M. Alves, Trad.). In C. Coll, J. Palácios, \& A. Marchesi (Eds.), Desenvolvimento psicológico e educação: Psicologia da educação (Vol. 2, pp.141-153). Porto Alegre: ArtMed. (Original publicado em 1993)

Escolano, A.M.C. \& Linhares, M.B.M. (1998). Estratégias de busca de informação em situação de resolução de problemas em crianças de primeira série do primeiro grau. [Resumos]. In Sociedade Brasileira de Psicologia (Ed.), Resumos de comunicações científicas, XVIII Reunião Anual de Psicologia (p.160). Ribeirão Preto: SBP.

Escolano, A.C.M. (2000). Avaliação cognitiva assistida em situação de resolução de problemas na predição do desempenho escolar de crianças de primeira série do primeiro grau. Dissertação de Mestrado não-publicada, Curso de Pós-Graduação em Psicologia, Faculdade de Filosofia, Ciências e Letras de Ribeirão Preto, Universidade de São Paulo. Ribeirão Preto, SP.

Ferrara, R.A., Brown, A.L., \& Campione, J.C. (1986). Children's learning and transfer of inductive reasoning rules: studies of proximal development. Child Development, 57, 1087-1099.

Ferreti, R.P. \& Butterfield, E.C. (1992). Intelligence related differences in the learning, maintenance and transfer of problem solving strategies. Intelligence, 16, 207-223.

Feuerstein, R, Rand, Y., \& Hoffman, M.B. (1979a). The dynamic assessment of retarded performers: the learning potential assessment device: Theory, instruments and techniques. Baltimore: University Park Press.

Feuerstein, R, Rand, Y., Hoffman, M, Hoffman, M., \& Miller, R. (1979b). Cognitive modifiability in retarded adolescents: effects of instrumental enrichment. American Journal of Mental Deficiency, 83, 539-550. 
Gera, A.A.S. (2001). Estratégias de perguntas de busca de informação na resolução de problemas em situação de avaliação assistida de crianças com queixa de dificuldade de aprendizagem. Dissertação de Mestrado não-publicada, Curso de Pós-Graduação em Psicologia, Faculdade de Filosofia, Ciências e Letras de Ribeirão Preto, Universidade de São Paulo. Ribeirão Preto, SP.

Linhares, M.B.M. (1995). Avaliação assistida: fundamentos, definição, características e implicações para a avaliação psicológica. Psicologia: Teoria e Pesquisa, 11, 23-31.

Linhares, M.B.M. (1996). Avaliação assistida em crianças com queixa de dificuldades de aprendizagem. Temas de Psicologia, 1, 17-32.

Linhares, M.B.M. (1998). Avaliação psicológica de aspectos cognitivos em crianças com queixa de dificuldades de aprendizagem. In C.A.R. Funayama (Ed.), Problemas de aprendizagem: enfoque multidisciplinar (pp.41-59). Ribeirão Preto: Legis Summa.

Linhares, M.B.M., Parreira, V.L.C., Marturano, A.C., \& Sant'Anna, S.C. (1993). Caracterização dos motivos de procura de atendimento infantil em um serviço de psicopedagogia clínica. Medicina, 26, 148-160.

Linhares, M.B.M., Santa Maria, M.R., Escolano, A.M.C., \& Gera, A.A.S. (1998). Avaliação cognitiva assistida: uma abordagem promissora na avaliação cognitiva de crianças. Temas em Psicologia, 7, 231-254.

Martin, E. \& Marchesi, A. (1995). Desenvolvimento metacognitivo e problemas de aprendizagem (A.M. Alves, Trad.). In C. Coll, J. Palácios, \& A. Marchesi (Eds.), Desenvolvimento psicológico e educação: necessidades educacionais e aprendizagem escolar (Vol. 3, pp. 24-35). Porto Alegre: ArtMed. (Original publicado em 1993)

Marturano, E.M., Loureiro, S.R., Linhares, M.B.M., \& Machado, V.L.S. (1997). Avaliação psicológica pode fornecer indicadores de problemas associados a dificuldades escolares? In E.M. Marturano, S.R. Loureiro, \& A.W. Zuardi (Eds.), Estudos em Saúde Mental (pp.11-47). Ribeirão Preto: Comissão de PósGraduação em Saúde Mental-FMRP/USP.

Mentis, M. (1997). Aprendizagem mediada dentro e fora da sala de aula (J.F. Azevedo, Trad.). São Paulo: Instituto Pieron de Psicologia Aplicada.

Peres, V.L.A. (1997). Triagem psicológica grupal: procedimentos e resultados obtidos com lista de espera de crianças, adolescentes e adultos, em uma clínica-escola de Psicologia. Paidéia - Cadernos de Psicologia e Educação da Faculdade de Filosofia, Ciências e Letras de Ribeirão Preto,12/13, 63-76.
Rueda, R. (1996). Desempenho assistido no ensino da escrita a estudantes com distúrbios de aprendizagem (F.A. Tesseler, Trad.). In L.C. Moel (Ed.), Vygotsky e a educação: implicações pedagógicas da psicologia sócio-histórica (pp.393-416). Porto Alegre: ArtMed.

Rutter, M. (1987). Psychological resilience and protective mechanisms. American Journal of Orthopsychiatric, 57, 316-331. Santa Maria, M.R. \& Linhares, M.B.M. (1999). Avaliação cognitiva assistida de crianças com indicação de dificuldades de aprendizagem escolar e deficiência mental. Psicologia: Reflexão E Crítica, 12, 395-417.

Short, E.J., Cuddy, C.L., Friebert, S.E., \& Schatschneider, C.W. (1990). The diagnostic and educational utility of thinking aloud during problem solving. In H.L. Swanson \& B. Keogh (Eds.), Learning disabilities: theoretical and research issues. Hillsdale, New Jersey: Erlbaum.

Swanson, H.L. (1995). Effects of dynamic testing on the classification of learning disabilities: the predictive and discriminant validity of the Swanson - cognitive processing test (S-CPT). Journal of Psychoeducational Assessment, 13, 204229

Vygotsky, L.S. (1988). A formação social da mente: o desenvolvimento dos processos psicológicos superiores (J.C. Neto, L.S.M. Barreto, \& S.C. Afeche, Trads.). São Paulo: Martins Fontes.

Weiss, M.L. (1992). Psicopedagogia clínica: uma visão diagnóstica. Porto Alegre: ArtMed. 